\title{
Recent Progress of In-Fiber Integrated Interferometers
}

\author{
Libo YUAN \\ Photonics Research Center, College of Science, Harbin Engineering University, Harbin 150001, P. R. China \\ *Corresponding author: Libo YUAN_ Email: lbyuan@vip.sina.com
}

\begin{abstract}
A brief review of recent progress made in a range of in-fiber integrated interferometers for measuring is presented, with particular attention paid to the multi-core based in-fiber integrated techniques, which have the potential to be exploited in a variety of wide applications.
\end{abstract}

Keywords: Optical fiber, multi-core fiber, fiber optic sensor, fiber optic interferometer

\section{Introduction}

Fiber optic interferometric structures have been largely explored in sensing due to their high sensitivity that they exhibit on the measurement of a broad range of parameters [1]. Independently of their alternative topological configurations by Michelson, Mach-Zehnder, Fabry-Perot, and Sagnac, etc. - different implementations have been considered aiming at to optimize the device performance for a particular set of measurands. The major part of this review is concerned with the developments related to multi-core fiber based in-fiber integrated interferometers and its applications to sensors. The basic fiber-optic interferometric sensing elements are conceptually the same as those described previously in the literatures [2]. However, in most sensor applications it is desirable that the fiber optic interferometric sensor is more compact and reliable. For this purpose, several techniques have been developed to build interferometer inside a string of fiber [3-4] called in-fiber integrated interferometers. The group of in-fiber integrated interferometers where the interferometric phase difference is built up referring to the difference in the effective refractive index of different fiber modes [5-8], the difference in the optical path $[9,10]$ as well as the difference in multi-core of one fiber $[5,11,12]$, have been widely researched in the context of environmental sensing, curvature sensing, and others. These structures are attractive for several reasons, including small size and deployment flexibility, as well as the presence of reduced thermal sensitivity in view of usually small difference of the thermo-optic coefficients of the fiber core and cladding under concern. In this paper, a briefly overview of the development of multi-core fiber based in-fiber integrated interferometers is presented.

\section{Category of in-fiber integrated interferometers}

\subsection{Meaning of in-fiber integrated interferometer}

In order to distinguish the difference of in-fiber integrated interferometers from the normal fiber optic interferometric structures, here, the so-called in-fiber integrated interferometer refers in particular to that the main parts of the light beams, beam splitters (combiners), and reflective mirrors are integrated in one fiber. In other words, all the interferometric constructional components should be

Received: 3 September 2010 / Revised version: 20 September 2010

(C) The Authors(s) 2010.This article is published with open access at Springerlink.com 
put in a fiber.

\subsection{Category of in-fiber integrated interferometers}

Based on the definition of in-fiber integrated interferometer mentioned above, their alternative topological configurations are shown in Fig. 1. It may be classified as: 1) Fabry-Perot; 2) Fizeau; 3) Michelson; 4) Mach-Zehnder; 5) multi-beam Mach-Zehnder in-fiber integrated interferometers.

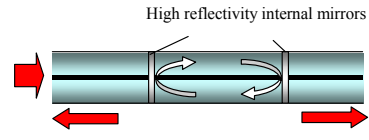

(a)
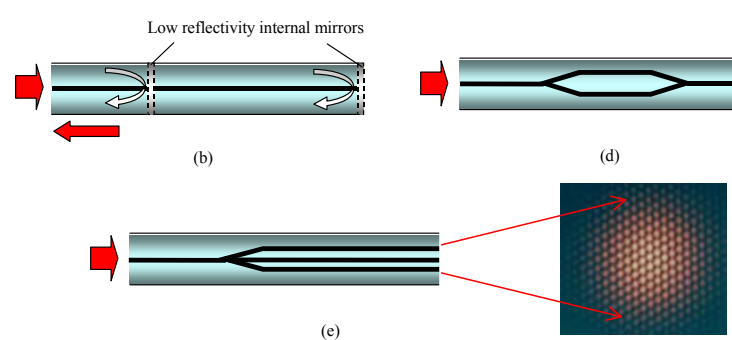

Fig. 1 In-fiber integrated interferometers: (a) in-fiber integrated Fabry-Perot interferometer, (b) in-fiber integrated Fizeau interferometer, (c) in-fiber integrated Michelson interferometer, (d) in-fiber integrated Mach-Zehnder interferometer, and (e) in-fiber integrated three-beams Mach-Zehnder interferometer.

\section{Two-core based in-fiber integrated interferometers}

The proposed in-fiber integrated interferometers are based on a two-beam element formed from a two-core fiber. A compact in-fiber integrated Michelson and Mach-Zehnder interferometer was easily implemented from the fused-tapering single-mode fiber to two-core fiber coupling technique [11]. The experimental setup is shown in Fig. 2. In the case of Michelson configuration (Fig. 2 (a)), a segment two-core fiber was fused with the standard single mode fiber and tapered at the splicing point, and at the end face of the two-core fiber a dielectric mirror was deposited directly on it. The light from a LD light source was launched into the fiber and passing though a 3-dB coupler directly going to the in-fiber integrated Michelson interferometer. And the reflected signals from the mirror end of the two-core fiber were combined in the tapered zone and received by the photo detector. In this manner a Michelson interferometer was simply implemented from a single fiber. Similarly, in the case of Mach-Zehnder configuration, between two segments of standard single-mode fiber, a segment of two-core fiber has been used. Three segments of fiber were fused together and tapered at two splice pinots, then the so-called in-fiber integrated Mach-Zehnder interferometer has been built, as shown in Fig. 2 (b).

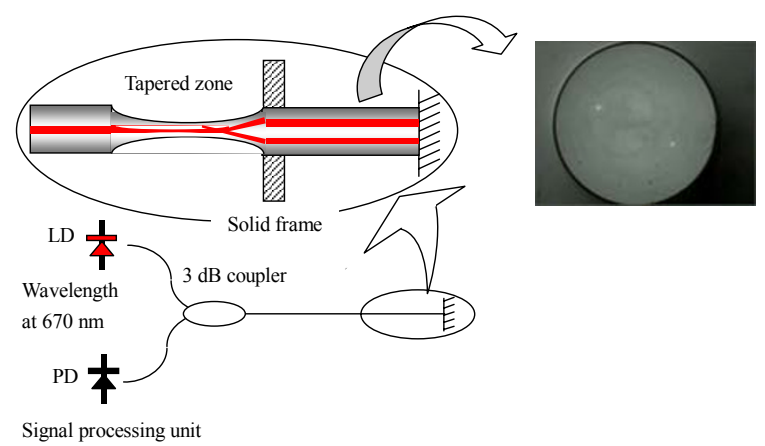

(a)

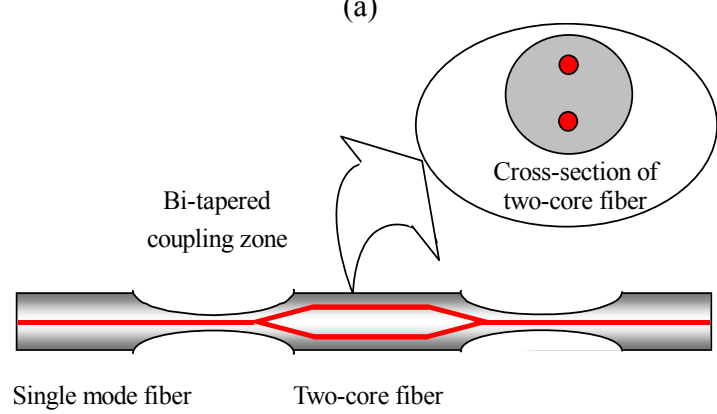

(b)

Fig. 2 In-fiber integrated compact Michelson and Mach-Zehnder interferometers built with two-core fiber: (a) in-fiber integrated Michelson interferometer and (b) in-fiber integrated Mach-Zehnder interferometer.

In order to establish the basic equations relating the optical path change induced in the two-core fiber to the fiber curvature, it is assumed that the fiber is a homogeneous and isotropic fused silica rod with radius $r$. For the case of weak curvature, i.e. the 
curvature radius $R$ is much larger than $r$. A simple analysis model can be established as shown in Fig. 3. And the optical phase differential of the two cores can be expressed as

$$
\delta \phi=\frac{k_{0} n L d}{R}\left[1-\mathrm{c}_{2} \frac{n^{2}}{2}\right] \cos \left[\theta_{0}+\frac{\pi}{4}+\theta(z)\right]
$$

where $k_{0}=2 \pi / \lambda$ is the wave number and $\lambda$ is the wavelength, $n$ is the refractive index of the fiber core, $L$ represents the length of the two-core fiber, $d$ is the distance between the two cores, $c_{2}=0.204$ is a constant for silica fiber, $\theta(z)$ is the twisting angle of the two-core fiber.

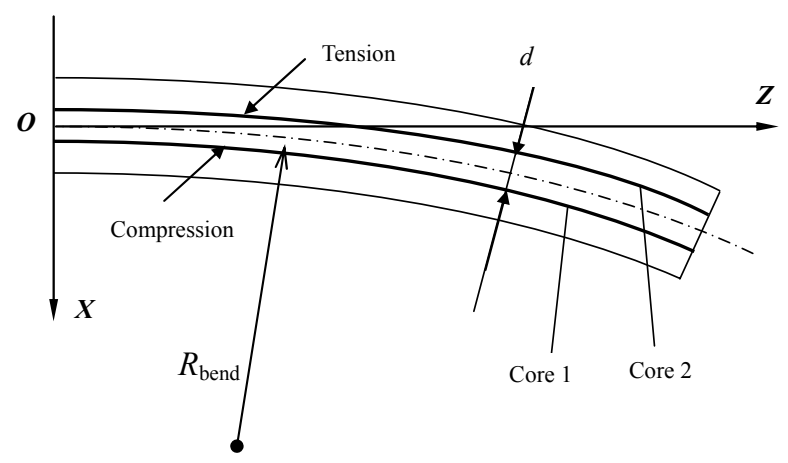

Fig. 3 Working principle of the two-core fiber bending sensor.

The two-core based in-fiber integrated interferometers could be wildly used for sensing, such as a displacement sensor, an accelerometer, and a vibration sensor. Unlike many other sensors, our sensors would be independent of environmental temperature and pressure changes because both arms of the interferometers would be affected equally by such changes. A bending sensor, for example, is described in Fig. 3, from which a curvature can be measured by the optical phase difference of the two cores. One application of the in-fiber integrated Mach-Zehnder interferometer as an integrated optical switch is reported in [13]. By attaching the two-core fiber based in-fiber integrated Mach-Zehnder interferometer on a cantilever beam, the switching characteristics has been investigated, as shown in Fig. 4.

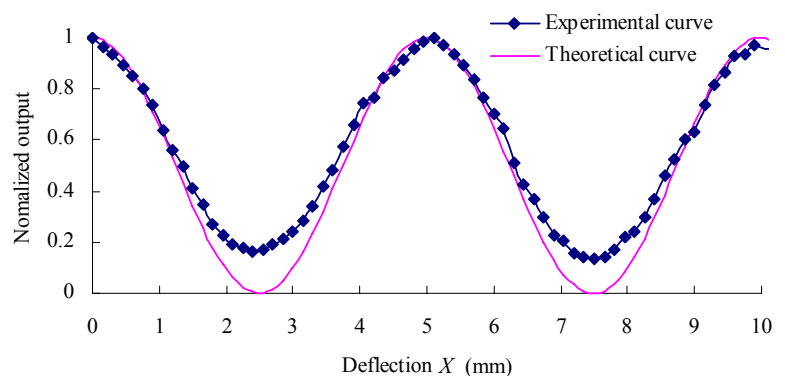

Fig. 4 Experimental characteristic curve of the in-fiber optic switch.

\section{Three-core and four-core based in-fiber integrated interferometers}

Multi-beam Mach-Zehnder interferometers by using three-core or four-core fiber as the sensing elements have been proposed and demonstrated [11]. The three-core or four-core fiber acts as a three-beam or four-beam interferometer in which phase differences is a function of curvature, twisting angle, and the variation of temperature in the plane containing the cores results in the shift of the far-field interferometric grid pattern. The relationship between the far-field grid pattern shift and the multi-parameters has been established; the parameters related with the optical phase differential of each core to the centre of the four-core fiber can be expressed as [11]

$$
\begin{aligned}
& \delta \phi_{m}=\frac{\sqrt{2} k_{0} n L d}{2}\left\{\left[1-\mathrm{c}_{2} \frac{n^{2}}{2}\right] \frac{1}{R}+A(T)\left[\alpha_{f}+C_{T}\right]\right\} \\
& \times \cos \left[\theta_{0}+(2 m-1) \frac{\pi}{4}+\theta(z)\right], \quad\left\{\begin{array}{c}
m=1,2,3 \\
\text { or } \\
m=1,2,3,4
\end{array}\right.
\end{aligned}
$$

where $k_{0}=2 \pi / \lambda$ is the wave number and $\lambda$ is the wavelength, $n$ is the refractive index of the fiber core, $L$ represents the length of the multi-core fiber, $A(T)$ is a constant related with the temperature gradient near by the fiber core, $C_{T}$ and $\alpha_{f}$ represent the temperature coefficient and the coefficient of thermal expansion for the four-core fiber respectively. For standard SMF-28 fiber, the temperature coefficient $C_{T}$ is, respectively, $0.762 \times 10^{-5} /{ }^{\circ} \mathrm{C}$ at $1300 \mathrm{~nm}$ and $0.811 \times 10^{-5} /{ }^{\circ} \mathrm{C}$ 
at $1550 \mathrm{~nm}$ [14]. The thermal expansion coefficient of the optical fiber is $\alpha_{f}=5.5 \times 10^{-7} /{ }^{\circ} \mathrm{C}$.

In order to get the information of three-core or four-core fiber's curvature, twisting angle as well as ambient temperature gradient value related with the far-field interferogram, a laser diode at wavelength $650 \mathrm{~nm}$ has been used in our experiments. For the three-core fiber, the internal distance between the neighbor cores was measured with the average value as $53 \mu \mathrm{m}$, shown in Fig. 5, while, for the four-core fiber, it was measured with the average value as $18 \mu \mathrm{m}$. The distance between the output fiber end and the CCD detection surface was measured as $D=3000 \mu \mathrm{m}$, shown in Fig. 6 (a). The simulation result is plotted in Fig. 6 (c). The interferogram grid pattern of the far field recorded by CCD camera is shown in Figs. 5 and 6 (d). The variation of parameter such as bending, twisting angle, and temperature gradient can be measured by accounting the shift of the grid pattern [11].

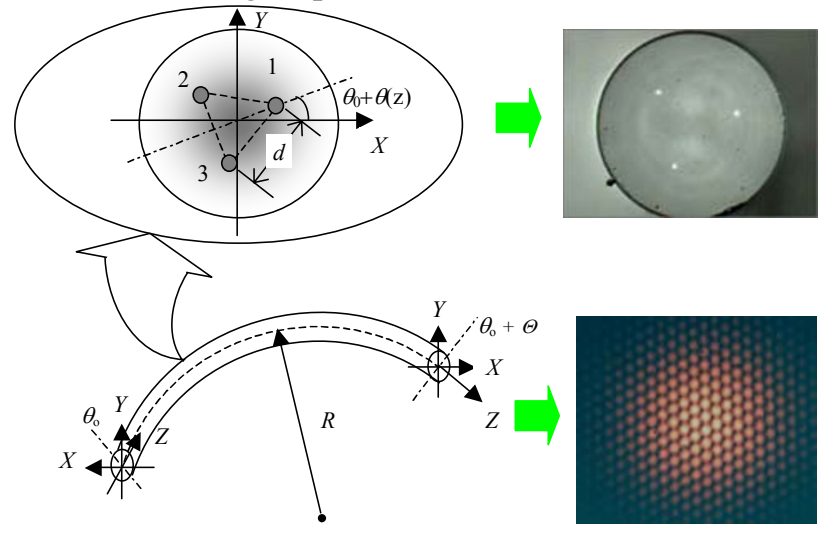

Fig. 5 Bend induced strain in the three-core fiber.

Optical $3 \mathrm{D}$ non-contact profilometry has been widely used for $3 \mathrm{D}$ sensing, mechanical engineering, machine vision, intelligent robots control, industry monitoring, biomedicine, dressmaking, etc. Based on the three-core fiber, a three-beam integrated Mach-Zehnder interferometer is made as shown in Fig. 1(e) and the output far field distribution formed an optic square (Fig. 7(a)) or hexagon (Fig. 5) grid interferogram. By using this technique, a shape mapping approach has been proposed and demonstrated in [15]. Theoretically, the three-core fiber coherence optical field intensity distribution generates a square or hexagon grid pattern.

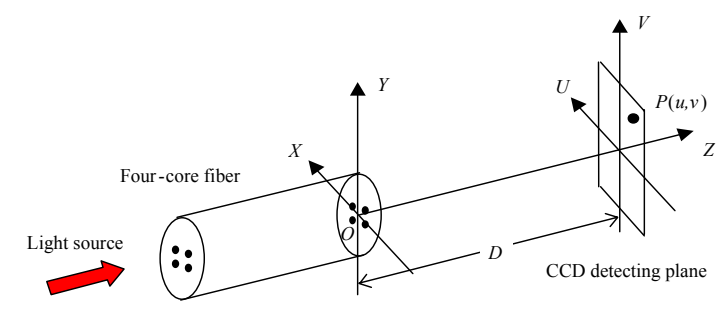

(a)

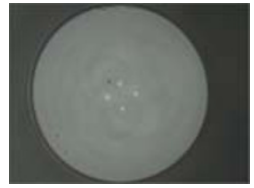

(b)

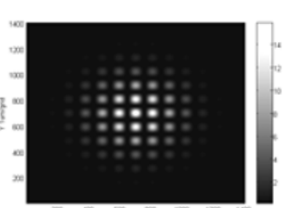

(c)

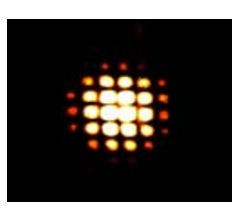

(d)
Fig. 6 Four-core fiber far field interferogram grid pattern: (a) experimental setup, (b) cross-section of four-core fiber, (c) theoretical simulation result, and (d) experimental result.

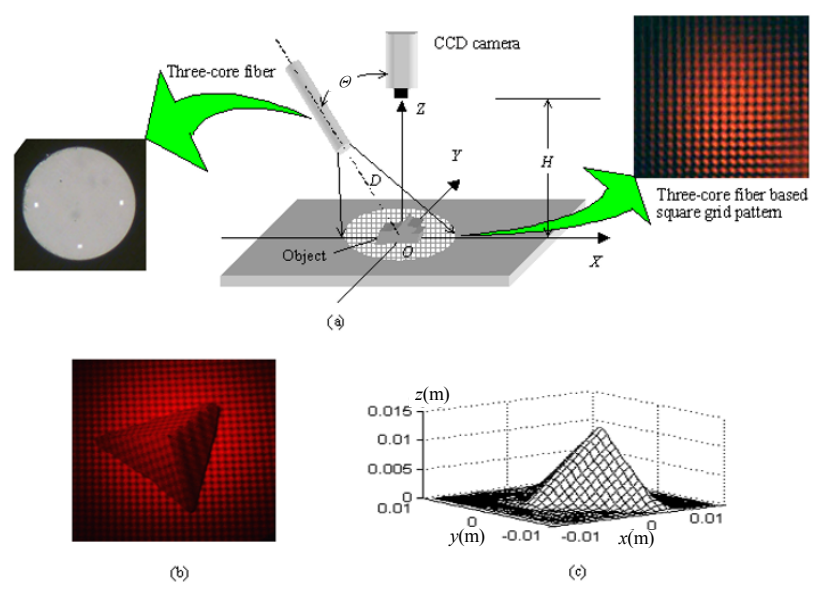

Fig. 7 Shape mapping experimental setup, in which the square grid pattern is generated by a special designed three-core fiber and the three cores of three-core fiber are located at the apex point of a right angle triangle: (a) experimental setup of the three-core fiber square grid pattern based shape-mapping system, (b) modulated grid pattern by a triangle pyramid, and (c) experimental results of the triangle pyramid reconstruction.

By using the 2 D Fourier transform methodology, the object surface can be reconstructed from the modulated fiber optic grid interferogram pattern. The shape mapping method, as shown in Fig. 7, is illustrated by a $3 \mathrm{D}$ shape measurement of uniformly 
optical square grid pattern modulated by triangle pyramid. In the case given by Fig. 7, the orthogonal phase data fusion method can be used in our experiment, which could greatly improve the accuracy of the $3 \mathrm{D}$ shape measurement results. It greatly simplifies the holographic interferometry system because the carrier square grid interferogram can be conveniently generated without the use of excessive auxiliary components or sophisticated experimental devices, and moreover, it can be used in very narrow places due to its small size. The advantage of three-core fiber based in-fiber integrated optic grid interferogram generation method used here is a simplified way to experimental realization.

\section{Conclusions}

In conclusion, based on the multi-core (in our case, two-core, three-core, and four-core) fiber, compact and stable in-fiber integrated Michelson and Mach-Zehnder interferometers as basic sensing device have been realized. The smart structures related parameters such as bending or curvature, twisting and temperature gradient can be measured by using the multi-core fiber. The small object shape also can be mapped by using the three-core fiber based three-beam integrated Mach-Zehnder interferometer. A lot of fiber optic sensors may be built up based on the in-fiber integrated interferometers, such as displacement sensor, curvature sensor, compact accelerator, and vibration sensor. It is shown that the multi-core fiber could be used as multi-parameter sensors and has the potential applications in a variety of wide applications.

\section{Acknowledgment}

This work was partially supported by the National Nature Science Foundation of China, under grant number 60877046, 60707013 and 60807032, to the Harbin Engineering University.

\section{References}

[1] D. A. Jackson, "Monomode optical fiber interferometers for precision measurement," J. Phys.
E: Sci. Instrum., vol. 18, no. 12, pp. 981-1001, 1985.

[2] D. A. Jackson, "Recent progress in monomode fiber-optic sensors," Meas. Sci. Technol., vol. 5, no. 6, pp. 621-638, 1994.

[3] X. Daxhelet, J. Bures, and R. Maciejko, "Temperature-independent all-fiber modal interferometer," Optical Fiber Technology, vol. 1, no. 4, pp. 373-376, 1995.

[4] O. Duhem, J. F. Henninot, and M. Douay, "Study of in fiber Mach-Zehnder interferometer based on two spaced 3-dB long period gratings surrounded by a refractive index higher than that of silica," Optics Communications, vol. 180, no. 4-6, pp. 255-262, 2000.

[5] L. B. Yuan, J. Yang, Z. H. Liu, and J. X. Sun, "In-fiber integrated Michelson interferometer," Optics Letters, vol. 31, no. 18, pp. 2692-2694, 2006.

[6] T. A. Eftimov and W. J. Bock, "Sensing with a LP01-LP02 intermodal interferometer," Journal of Lightwave Technology, vol. 11, no. 12, pp. 2150-2156, 1993.

[7] A. Kumar, N. K. Goel, and R. K. Varshney, "Studies on a few-mode fiber-optic strain sensor based on LP01-LP02 Mode interference," Journal of Lightwave Technology, vol. 19, no. 3, pp. 358-362, 2001.

[8] D. Kacik, I. Turek, I. Martincek, J. Canning, N. A. Issa, and K. Lyytikainen, "Intermodal interference in a photonic crystal fiber," Optics Express, vol. 12, no. 15 , pp. $3465-3470,2004$

[9] J. Villatoro, V. P. Minkovich, and D. Monzon-Hernandez, "Compact modal interferometer built with tapered microstructured optical fiber," IEEE Photonics Technology Letters, vol. 18, no. 11, pp. 1258-1260, 2006.

[10] P. L. Swart, "Long-period grating Michelson refractometric sensor," Measurement Science and Technology, vol. 15, no. 8, pp. 1576-1580, 2004.

[11] L. B. Yuan and X. Wang, "Four-beam single fiber optic interferometer and its sensing characteristics," Sensors and Actuators, vol. 138, no. 1, pp. 9-15, 2007.

[12] L. B. Yuan, Z. H. Liu, and J. Yang, "Coupling characteristics between single core fiber and multi-core fiber," Optics Letters, vol. 31, no. 22, pp. 3237-3239, 2006.

[13] Z. H. Liu, J. Yang, Z. F. Wu, and L. B. Yuan, "A novel in-fiber optical switch based on two-core optical fiber," in SPIE Proc., vol. 6838, pp. 68380F, 2008.

[14] L. B. Yuan, "Effect of temperature and strain on fiber optic refractive index," Acta Optica Sinica, vol. 17, no. 12, pp. 1713-1717, 1997 (in chinese).

[15] L. B. Yuan, J. Yang, C. Y. Guan, Q. Dai, and F. J. Tian, "Three-core fiber based shape sensing application," Optics Letters, vol. 33, no. 6, pp. 578-581, 2008. 